\title{
Rule-based target differentiation and position estimation based on infrared intensity measurements
}

\author{
Tayfun Aytaç \\ Billur Barshan \\ Bilkent University \\ Department of Electrical Engineering \\ 06800 Bilkent \\ Ankara, Turkey \\ E-mail: billur@ee.bilkent.edu.tr
}

\begin{abstract}
This study investigates the use of low-cost infrared sensors in the differentiation and localization of target primitives commonly encountered in indoor environments, such as planes, corners, edges, and cylinders. The intensity readings from such sensors are highly dependent on target location and properties in a way that cannot be represented in a simple manner, making the differentiation and localization difficult. We propose the use of angular intensity scans from two infrared sensors and present a rule-based algorithm to process them. The method can achieve position-invariant target differentiation without relying on the absolute return signal intensities of the infrared sensors. The method is verified experimentally. Planes, 90-deg corners, 90-deg edges, and cylinders are differentiated with correct rates of $90 \%, 100 \%, 82.5 \%$, and $92.5 \%$, respectively. Targets are localized with average absolute range and azimuth errors of $0.55 \mathrm{~cm}$ and $1.03 \mathrm{deg}$. The demonstration shows that simple infrared sensors, when coupled with appropriate processing, can be used to extract a significantly greater amount of information than they are commonly employed for. (c) 2003 Society of Photo-Optical Instrumentation Engineers. [DOI: 10.1117/1.1570428]
\end{abstract}

Subject terms: pattern recognition and feature extraction; position estimation; target differentiation and localization; infrared sensors; optical sensing.

Paper 020383 received Sep. 4, 2002; revised manuscript received Oct. 28, 2002; accepted for publication Nov. 18, 2002.

\section{Introduction}

Target differentiation and localization are of importance for intelligent systems that need to interact with and autonomously operate in their environment. In this paper, we consider the use of infrared sensors for this purpose. Infrared sensors are inexpensive, practical, and widely available devices. Simple range estimates obtained with infrared sensors are not reliable, because the return signal intensity depends on both the geometry and the surface properties of the target. On the other hand, from single intensity measurements it is not possible to deduce the geometry and surface properties of the target without knowing its distance and angular location. In this study, we propose a scanning mechanism and a rule-based algorithm based on two infrared sensors to differentiate targets independently of their locations. The proposed method has the advantage of minimal storage requirements, since the information necessary to differentiate the targets is completely embodied in the decision rules.

Application areas of infrared sensing include robotics and automation, process control, remote sensing, and safety and security systems. More specifically, infrared sensors have been used in simple object and proximity detection, counting, ${ }^{1,2}$ distance and depth monitoring, ${ }^{3}$ floor sensing, position control, ${ }^{4}$ obstacle and collision avoidance, ${ }^{5}$ and machine vision systems. ${ }^{6}$ Infrared sensors are used in door detection, ${ }^{7}$ mapping of openings in walls, ${ }^{8}$ monitoring doors and windows of buildings and vehicles, and light curtains for protecting an area. In Ref. 9, an automated guided vehicle detects unknown obstacles by means of an "electronic stick" consisting of infrared sensors, using a strategy similar to that adopted by a blind person. In Ref. 10 , infrared sensors are employed to locate edges of doorways in a complementary manner with sonar sensors. Other researchers have also dealt with the fusion of information from infrared and sonar sensors ${ }^{11,12}$ and from infrared and radar systems. ${ }^{13,14}$ In Ref. 15, infrared proximity sensing for a robot arm is discussed. Following this work, Ref. 5 describes a robot arm completely covered with an infrared skin sensor to detect nearby objects. In another study, ${ }^{16}$ the properties of a planar surface at a known distance have been determined using the Phong illumination model, and using this information, the infrared sensor employed has been modeled as an accurate rangefinder for surfaces at short ranges.

Reference 17 also deals with determining the range of a planar surface. By incorporating the optimal amount of additive noise in the infrared range measurement system, the authors were able to improve the system sensitivity and extend the operating range of the system.

A number of commercially available infrared sensors are evaluated in Ref. 18. References 19 and 20 describe a passive infrared sensing system that identifies the locations of the people in a room. Infrared sensors have also been used for automated sorting of waste objects made of different materials. $^{21,22}$ 
However, to the best of our knowledge, no attempt has been made to differentiate and estimate the position of several kinds of targets using infrared sensors. This represents the extraction of a significantly greater amount of information from such simple sensors than in earlier work.

Most work on pattern recognition involving infrared deals with recognition or detection of features or targets in conventional two-dimensional images. Examples of work in this category include face identification, ${ }^{23}$ automatic target recognition, ${ }^{24}$ automatic vehicle detection, ${ }^{25}$ remote sensing, ${ }^{26}$ detection and identification of targets in background clutter, ${ }^{27}$ and automated terrain analysis. ${ }^{28}$ We note that the position-invariant pattern recognition and position estimation achieved in this paper are different from such operations performed on conventional images in that here we work not on direct "photographic" images of the targets obtained by some kind of imaging system, but rather on angular intensity scans obtained by rotating a pair of sensors. The targets we differentiate are not patterns in a twodimensional image whose coordinates we try to determine, but rather objects in space, exhibiting depth, whose position with respect to the sensing system we need to estimate. For this reason, position-invariant differentiation and localization are achieved with an approach quite different than those employed for invariant pattern recognition and localization of conventional images (for instance, see Ref. 29).

In Ref. 30, we considered processing information provided by a single infrared sensor using least-squares and matched-filtering methods, comparing observed scans with previously stored reference scans. In this paper, we consider processing information from a pair of sensors using a rule-based approach. The advantages of a rule-based approach are shorter processing times, greater robustness to noise, and minimal storage requirements in that it does not require storage of any reference scans: the information necessary to differentiate the targets is completely embodied in the decision rules. Examples of related approaches with sonar sensors may be found in Refs. 31 and 32.

This paper is organized as follows: In Sec. 2, we describe the target differentiation and localization process employed. Section 3 provides experimental verification of the approach presented in this paper. Concluding remarks are made and directions for future research are provided in the last section.

$$
E
$$

plane

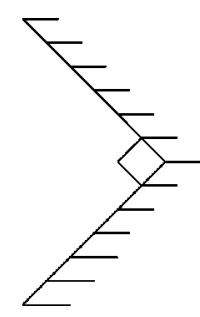

corner

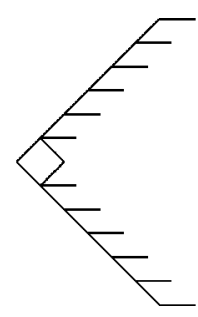

edge

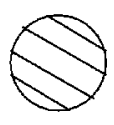

Fig. 1 Target primitives used in the experiment.

\section{Target Differentiation and Localization}

The infrared sensor ${ }^{33}$ used in this study consists of an emitter and detector and works with $20-$ to $28-\mathrm{V}$ dc input voltage; it provides an analog output voltage proportional to the measured intensity reflected off the target. The detector window is covered with an infrared filter to minimize the effect of ambient light on the intensity measurements. Indeed, when the emitter is turned off, the detector reading is essentially zero. The sensitivity of the device can be adjusted with a potentiometer to set the operating range of the system. The range, azimuth, geometry, and surface parameters of the target affect the intensity readings of the infrared sensors.

The target primitives employed in this study are a plane, a 90-deg corner, a 90-deg edge, and a cylinder of radius 4.8 $\mathrm{cm}$, whose cross sections are given in Fig. 1. The horizontal extent of all targets other than the cylinder is large enough that they can be considered infinite and thus edge effects need not be considered. They are made of wood, each with a height of $120 \mathrm{~cm}$. Our method is based on angularly scanning the target over a certain angular range. We use two infrared sensors horizontally mounted on a 12 -in. rotary table ${ }^{34}$ with a center-to-center separation of $11 \mathrm{~cm}$ (Fig. 2). Targets are scanned from -60 to 60 deg in 0.15deg increments, and the mean of 100 samples is calculated at each position of the rotary table. The targets are situated at ranges varying between 20 and $65 \mathrm{~cm}$. The outputs of the infrared sensors are multiplexed to the input of an 8-bit microprocessor-compatible analog-to-digital converter chip having a conversion time of $100 \mu \mathrm{s}$.

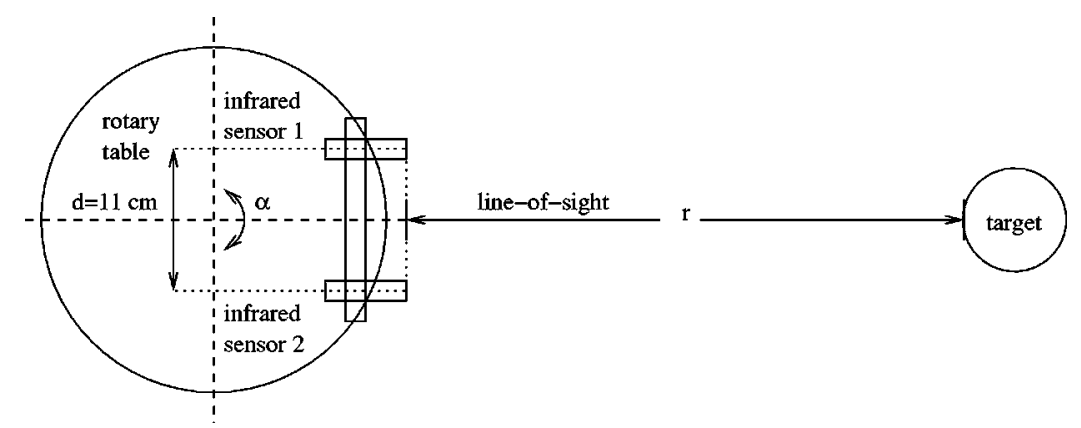

Fig. 2 The experimental setup. Both the scan angle $\alpha$ and the target azimuth $\theta$ are measured counterclockwise from the horizontal axis. 


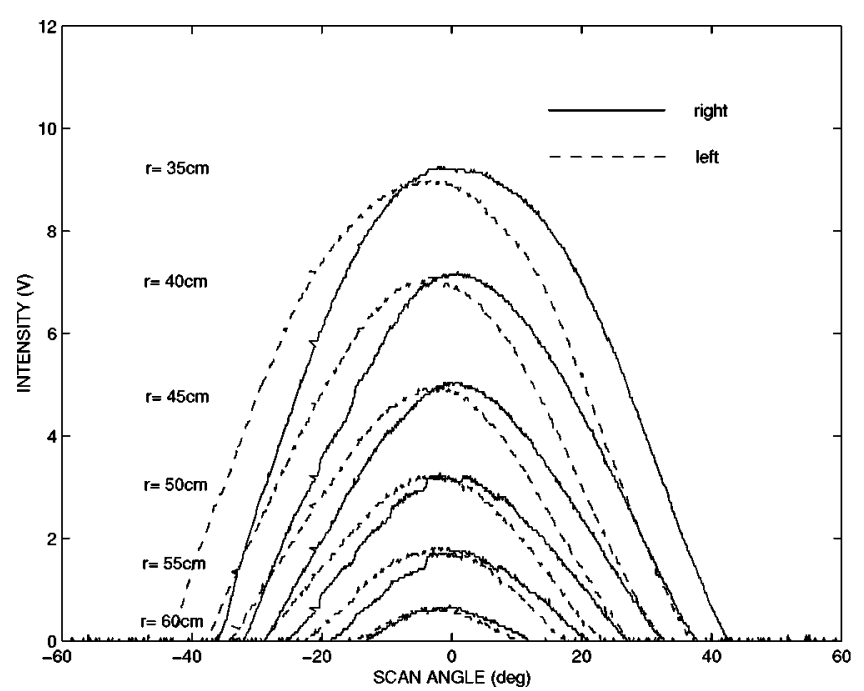

(a)

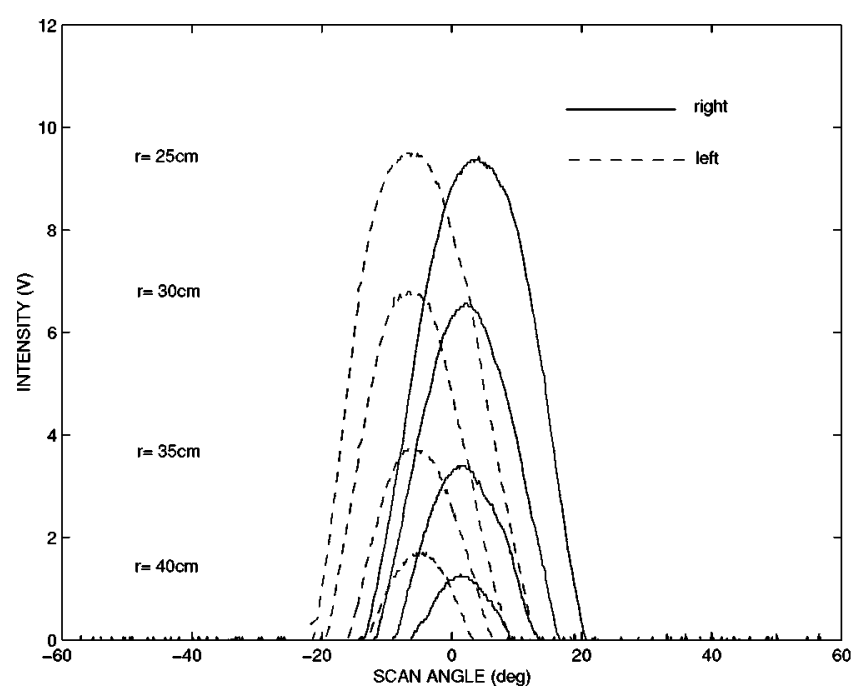

(c)

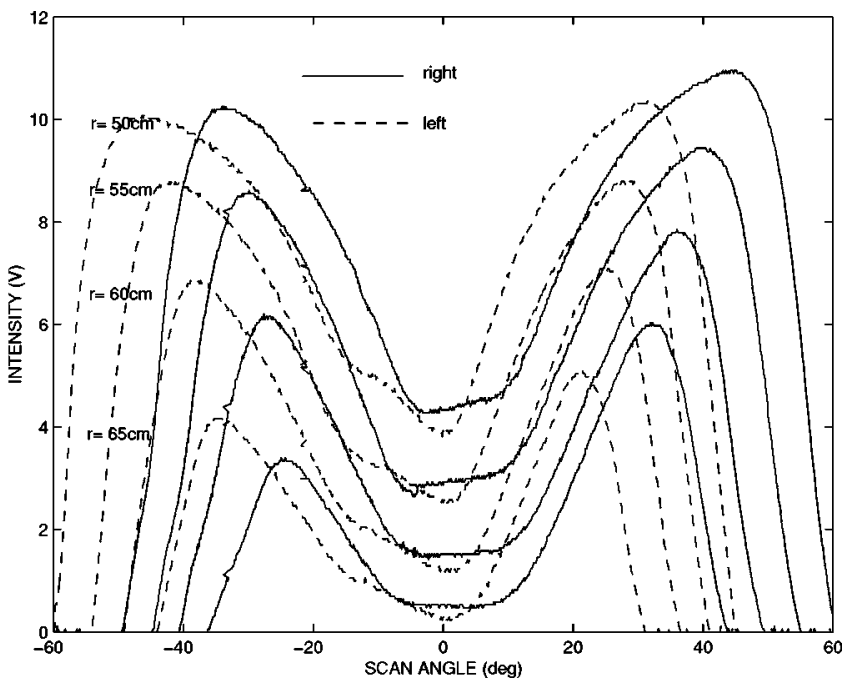

(b)

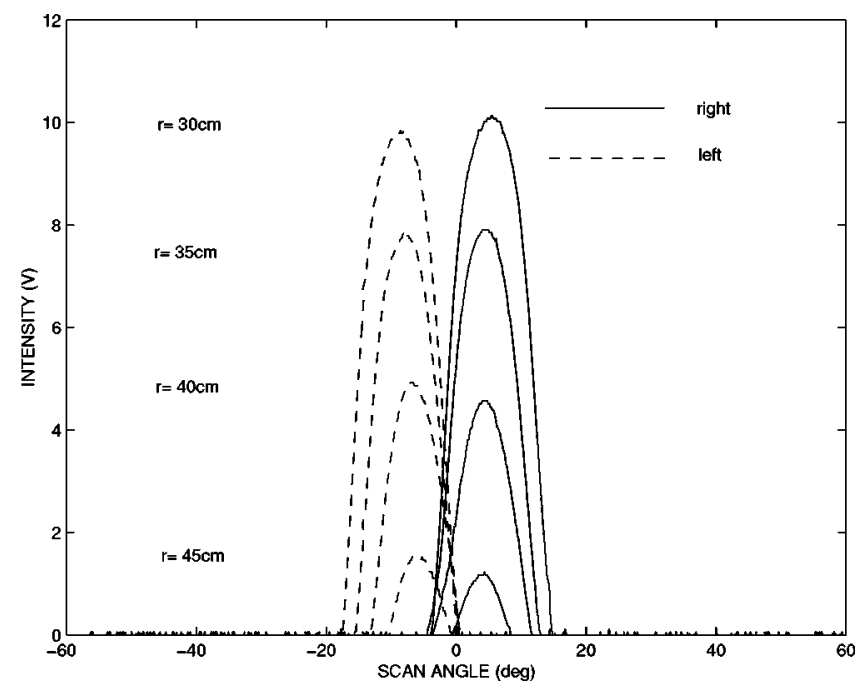

(d)

Fig. 3 Intensity-versus-scan-angle characteristics for various targets along the line of sight of the experimental setup. (a) Plane; (b) corner; (c) edge; (d) cylinder.

Some sample scan patterns obtained from the targets are shown in Fig. 3. Based on these patterns, it is observed that the return signal intensity patterns for a corner, which have two maxima and a single minimum (a double-humped pattern), differ significantly from those of other targets, which have a single maximum [Fig. 3(b)]. The double-humped pattern is a result of the two orthogonal planes constituting the corner. Because of these distinctive characteristics, the corner differentiation rule is employed first. We check if the scan pattern has two humps or not. If so, it is a corner. The average of the angular locations of the dips in the middle of the two humps for the left and right infrared sensors provides an estimate of the angular location of the corner.

If the target is found not to be a corner, we next check whether it is a plane or not. As seen in Fig. 3(a), the difference between the angular locations of the maximum readings for the planar targets is significantly smaller than for other targets. Planar targets are differentiated from other targets by examining the absolute difference of the angle values at which the two intensity patterns have their maxima. If the difference is less than an empirically determined reference value, then the target is a plane; otherwise, it is either an edge or a cylinder. (In the experiments, we have used a reference value of $6.75 \mathrm{deg}$.) The azimuth estimation of planar targets is accomplished by averaging the angular locations of the maxima of the two scans associated with the two sensors.

Notice that the above (and the following) rules are designed to be independent of those features of the scans that vary with range and azimuth, so as to enable positioninvariant recognition of the targets. In addition, the proposed method has the advantage that it does not require storage of any reference scans, since the information necessary to differentiate the targets is completely embodied in the decision rules.

If the target is not a plane either, we next check whether it is an edge or a cylinder. The intensity patterns for the edge and the cylinder are given in Figs. 3(c) and 3(d). They 
Table 1 Target confusion matrix (P: plane; C: corner; E: edge; CY: cylinder).

\begin{tabular}{lrrrrr}
\hline \hline & \multicolumn{4}{c}{ Differentiation result } & \\
\cline { 2 - 5 } Target & P & C & E & CY & Total \\
\hline P & 36 & - & 4 & - & 40 \\
C & - & 40 & - & - & 40 \\
E & 4 & - & 33 & 3 & 40 \\
CY & 3 & - & - & 37 & 40 \\
Total & 43 & 40 & 37 & 40 & 160 \\
\hline \hline
\end{tabular}

have shapes similar to those of planar targets, but the intersection points of the intensity patterns differ significantly from those of planar targets. In the differentiation between edges and cylinders, we employ the intensity value at the intersection of the two scans corresponding to the two sensors, divided by the maximum intensity value of the scans. (Because the maximum intensities of the right and left infrared scans are very close, the maximum intensity reading of either infrared sensor or their average can be used in this computation.) This ratio is compared with an empirically determined reference value to determine whether the target is an edge or a cylinder. If the ratio is greater than the reference value, the target is an edge; otherwise, it is a cylinder. (In our experiments, the reference value was 0.65.) If the scan patterns from the two sensors do not intersect, the algorithm cannot distinguish between a cylinder and an edge. However, this never occurred in our experiments. The azimuth estimate of edges and cylinders is also obtained by averaging the angular locations of the maxima of the two scans. Having determined the target type and estimated its azimuth, its range can also be estimated by using linear interpolation between the central values of the individual intensity scans given in Fig. 3.

\section{Experimental Verification of the Algorithm}

Using the experimental setup described in Sec. 2, the algorithm presented in that section was used to differentiate and estimate the position of a plane, a 90-deg corner, a 90-deg edge, and a cylinder of radius $4.8 \mathrm{~cm}$.

Based on the results for 160 experimental test scans (from 40 different locations for each target), the target confusion matrix shown in Table 1, which contains information about the actual and detected targets, is obtained. The average accuracy over all target types can be found by summing the correct decisions given along the diagonal of the confusion matrix and dividing this sum by the total number of test scans (160), resulting in an average accuracy of 91.3\% over all target types. Targets are localized within absolute average range and azimuth errors of $0.55 \mathrm{~cm}$ and $1.03 \mathrm{deg}$, respectively. The errors have been calculated by averaging the absolute differences between the estimated ranges and azimuths and the actual ranges and azimuths read off from the millimetric grid paper covering the floor of the experimental setup.

The percentage accuracy and confusion rates are presented in Table 2 . The second column of the table gives the percentage accuracy of correct differentiation of the target, and the third column gives the percentage of cases when
Table 2 Performance parameters of the algorithm (P: plane; C: corner; E: edge; CY: cylinder).

\begin{tabular}{lccc}
\hline \hline $\begin{array}{l}\text { Actual } \\
\text { target }\end{array}$ & $\begin{array}{c}\text { Correct diff. } \\
\text { rate (\%) }\end{array}$ & $\begin{array}{c}\text { Differen. } \\
\text { error I (\%) }\end{array}$ & $\begin{array}{c}\text { Differen. } \\
\text { error II (\%) }\end{array}$ \\
\hline P & 90 & 10 & 16.3 \\
C & 100 & 0 & 0 \\
E & 82.5 & 17.5 & 10.8 \\
CY & 92.5 & 7.5 & 7.5 \\
Overall & 91.25 & 8.75 & 8.65 \\
\hline \hline
\end{tabular}

one target was mistaken for another. The fourth column gives the total percentage of other target types that were mistaken for a particular target type. For instance, for the planar target $(4+3) / 43=16.3 \%$, meaning that targets other than planes are incorrectly classified as planes with a rate of $16.3 \%$.

Because the intensity pattern of a corner differs significantly from that of the rest of the targets, the algorithm differentiates corners accurately with a rate of $100 \%$. A target is never classified as a corner if it is actually not a corner. Edges and cylinders are the most difficult targets to differentiate.

\section{Conclusion}

In this study, differentiation and localization of commonly encountered targets or features such as planes, corners, edges, and cylinders is achieved using intensity measurements from inexpensive infrared sensors. We propose a scanning mechanism and a rule-based algorithm based on two infrared sensors to differentiate targets independently of their positions. We have shown that the resulting angular intensity scans contain sufficient information to identify several different target types and estimate their distance and azimuth. The algorithm is evaluated in terms of its correct target differentiation rate and its range and azimuth estimation accuracy.

A typical application of the demonstrated system would be in mobile robotics in surveying an unknown environment composed of such features or targets. Many artificial environments fall into this category. We plan to test and evaluate the developed system on a small mobile robot in our laboratory for map building in a test room composed of the primitive target types considered in this study.

The accomplishment of this study is that even though the intensity scan patterns are highly dependent on target location, and this dependence cannot be represented by a simple relationship, we achieve position-invariant target differentiation. By designing the decision rules so that they do not depend on those features of the scans that vary with range and azimuth, an average correct target differentiation rate of $91.3 \%$ over all target types is achieved, and targets are localized within average absolute range and azimuth errors of $0.55 \mathrm{~cm}$ and $1.03 \mathrm{deg}$, respectively. The proposed method has the advantage that it does not require storage of any reference scans, since the information necessary to differentiate the targets are completely embodied in the decision rules. The method also exhibits considerable robustness to deviations in geometry or surface properties of the 
targets, since the rule-based approach emphasizes structural features rather than the exact functional forms of the scans. The major drawback of the present method, as with all such rule-based methods, is that the rules are specific to the set of objects and must be modified for a different set of objects. Nevertheless, the rules we propose in this paper are of considerable practical value, since the set of objects considered in this paper is an important set consisting of the most commonly encountered features in typical indoor environments and therefore deserves a custom set of rules. (Differentiating this set of objects has long been the subject of investigations involving sonar sensors. ${ }^{35-38}$ )

In this paper, we have demonstrated differentiation of four basic target types having similar surface properties. Broadly speaking, the major effect of different materials and textures is to change the reflectivity coefficients of the objects. This in turn will primarily have the effect of modifying the amplitudes of the scans, with less effect on their structural forms. Therefore, the same general set of rules can be applied with minor modifications or mere adjustments of the parameters. Current work investigates the deduction of not only the geometry but also the surface properties of the target from its intensity scans without knowing its location.

\section{Acknowledgments}

This research was supported by TÜBITAK under BDP and 197E051 grants. The authors would like to thank the Department of Engineering Science of the University of Oxford for donating the infrared sensors.

\section{References}

1. K. Hashimoto, C. Kawaguchi, S. Matsueda, K. Morinaka, and N. Yoshiike, "People counting system using multisensing application," Sens. Actuators A 66(1-3), 50-55 (1998).

2. A. J. Hand, "Infrared sensor counts insects," Photonics Spectra 32(11), 30-31 (1998).

3. H. C. Wikle, S. Kottilingam, R. H. Zee, and B. A. Chin, "Infrared sensing techniques for penetration depth control of the submerged arc welding process," J. Mater. Process. Technol. 113(1-3), 228-233 (2001).

4. B. Butkiewicz, "Position control system with fuzzy microprocessor AL220," Lect. Notes Comput. Sci. 1226, 74-81 (1997).

5. V. J. Lumelsky and E. Cheung, "Real-time collision avoidance in teleoperated whole-sensitive robot arm manipulators," IEEE Trans. Syst. Man Cybern. 23(1), 194-203 (1993).

6. H. R. Everett, Sensors for Mobile Robots, Theory and Application, A. K. Peters, Ltd., 289 Linden St., Wellesley, MA (1995).

7. G. Beccari, S. Caselli, and F. Zanichelli, "Qualitative spatial representations from task-oriented perception and exploratory behaviors," Rob. Auton. Syst. 25(3/4), 147-157 (1998).

8. A. Warszawski, Y. Rosenfeld, and I. Shohet, "Autonomous mapping system for an interior finishing robot," J. Comput. Civ. Eng. 10(1), 67-77 (1996).

9. E. P. Lopes, E. P. L. Aude, J. T. C. Silveria, H. Serderia, and M. F. Martins, "Application of a blind person strategy for obstacle avoidance with the use of potential fields," in Proc. IEEE Int. Conf. on Robotics and Automation, Vol. 3, pp. 2911-2916, Seoul (2001).

10. A. M. Flynn, "Combining sonar and infrared sensors for mobile robot navigation," Int. J. Robot. Res. 7(6), 5-14 (1988).

11. H. M. Barberá, A. G. Skarmeta, M. Z. Izquierdo, and J. B. Blaya, "Neural networks for sonar and infrared sensors fusion," in Proc Third Int. Conf. on Information Fusion, Vol. 2, pp. 18-25, International Society of Information Fusion (ISIF) (2000).

12. A. M. Sabatini, V. Genovese, E. Guglielmelli, A. Mantuano, G. Ratti, and P. Dario, "A low-cost, composite sensor array combining ultrasonic and infrared proximity sensors," in Proc. IEEE/RSJ Int. Conf. on Intelligent Robots and Systems, pp. 120-126, Pittsburgh (1995).

13. B. Chen and J. K. Tugnait, "Multisensor tracking of a maneuvering target in clutter using IMMPDA fixed-lag smoothing," IEEE Trans. Aerosp. Electron. Syst. 36(3), 983-991 (2000).

14. Y. M. Chen and H. C. Huang, "Fuzzy logic approach to multisensor data association," Math. Comput. Simul. 52(5/6), 399-412 (2000).
15. E. Cheung and V. J. Lumelsky, "Proximity sensing in robot manipulator motion planning: system and implementation issues," IEEE Trans. Rob. Autom. 5(6), 740-751 (1989).

16. P. M. Novotny and N. J. Ferrier, "Using infrared sensors and the Phong illumination model to measure distances," in Proc. IEEE Int. Conf. on Robotics and Automation, pp. 1644-1649, Detroit (1999).

17. B. Andò and S. Graziani, "A new IR displacement system based on noise added theory," in IEEE Instrum. Meas. Technol. Conf., pp. 482485 (Budapest 2001).

18. L. Korba, S. Elgazzar, and T. Welch, "Active infrared sensors for mobile robots," IEEE Trans. Instrum. Meas. 43(2), 283-287 (1994).

19. K. Hashimoto, T. Tsuruta, K. Morinaka, and N. Yoshiike, "High performance human information sensor," Sens. Actuators A 79(1), 46-52 (2000)

20. N. Yoshiike, K. Morinaka, K. Hashimoto, M. Kawaguri, and S Tanaka, "360 degrees direction type human information sensor," Sens. Actuators A 77(3), 199-208 (1999).

21. P. J. de Groot, G. J. Postma, W. J. Melssen, and L. M. C. Buydens, "Validation of remote, on-line, near-infrared measurements for the classification of demolition waste," Anal. Chim. Acta 453(1), 117124 (2002).

22. D. M. Scott, "A 2-color near-infrared sensor for sorting recycled plastic waste," Meas. Sci. Technol. 6(2), 156-159 (1995).

23. P. J. Phillips, "Matching pursuit filters applied to face identification," IEEE Trans. Image Process. 7(8), 1150-1164 (1998).

24. P. V. Noah, M. A. Noah, J. Schroeder, and J. Chernick, "Background characterization techniques for target detection using scene metrics and pattern recognition," Opt. Eng. 30(3), 254-258 (1991).

25. I. Pavlidis, P. Symosek, B. Fritz, M. Bazakos, and N. Papanikolopoulos, "Automatic detection of vehicle occupants: the imaging problem and its solution," Mach. Vision Appl. 11(6), 313-320 (2000).

26. T. A. Berendes, K. S. Kuo, A. M. Logar, E. M. Corwin, R. M. Welch, B. A. Baum, A. Pretre, and R. C. Weger, "A comparison of paired histogram, maximum likelihood, class elimination, and neural network approaches for daylight global cloud classification using AVHRR imagery," J. Geophys. Res., [Atmos.] 104(D6), 6199-6213 (1999).

27. Y. S. Moon, T. X. Zhang, Z. R. Zuo, and Z. Zuo, "Detection of sea surface small targets in infrared images based on multilevel filter and minimum risk Bayes test," Int. J. Pattern Recognit. Artif. Intell. 14(7), 907-918 (2000).

28. B. Bhanu, P. Symosek, and S. Das, "Analysis of terrain using multispectral images," Pattern Recogn. 30(2), 197-215 (1997).

29. F. T. S. Yu and S. Yin, Eds., Selected Papers on Optical Pattern Recognition, SPIE Milestone Series, Vol. MS 156, SPIE Optical Engineering Press, Bellingham, WA (1999).

30. T. Aytaç and B. Barshan, "Differentiation and localization of targets using infrared sensors," Opt. Coтmun. 210(1-2), 25-35 (2002).

31. B. Barshan and R. Kuc, "Differentiating sonar reflections from corners and planes by employing an intelligent sensor," IEEE Trans. Pattern Anal. Mach. Intell. 12(6), 560-569 (1990).

32. B. Ayrulu and B. Barshan, "Identification of target primitives with multiple decision-making sonars using evidential reasoning," Int. J. Robot. Res. 17(6), 598-623 (1998).

33. Proximity Switch Datasheet, IRS-U-4A, Matrix Elektronik AG, Kirchweg 24, CH-5422 Oberehrendingen, Switzerland (1995).

34. RT-12 Rotary Positioning Table, Arrick Robotics, P.O. Box 1574, Hurst, Texas, 76053, www.robotics.com/rt12.html (2002).

35. R. Kuc and M. W. Siegel, "Physically-based simulation model for acoustic sensor robot navigation," IEEE Trans. Pattern Anal. Mach. Intell. PAMI-9(6), 766-778 (1987).

36. L. Kleeman and R. Kuc, "Mobile robot sonar for target localization and classification," Int. J. Robot. Res. 14(4), 295-318 (1995).

37. J. J. Leonard and H. F. Durrant-Whyte, "Mobile robot localization by tracking geometric beacons," IEEE Trans. Rob. Autom. 7(3), 376-382 (1991).

38. O. Bozma and R. Kuc, "Building a sonar map in a specular environment using a single mobile sensor," IEEE Trans. Pattern Anal. Mach. Intell. 13(12), 1260-1269 (1991).

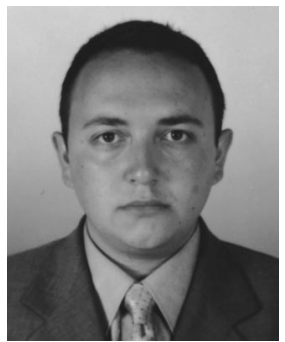

Tayfun Aytaç received a BS degree in electrical engineering from Gazi University, Ankara, Turkey in 2000 and a MS degree in electrical engineering from Bilkent University, Ankara, Turkey in 2002. He is currently working towards his $\mathrm{PhD}$ degree in the same department. His current research interests include intelligent sensing, optical sensing, pattern recognition, sensor data fusion, target differentiation, and sensorbased robotics. 


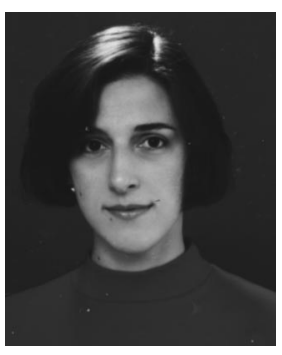

Billur Barshan received BS degrees in both electrical engineering and physics from Boğaziçi University, Istanbul, Turkey, and $\mathrm{MS}$ and $\mathrm{PhD}$ degrees in electrical engineering from Yale University, New Haven, Connecticut, in 1986, 1988, and 1991, respectively. Dr. Barshan was a research assistant at Yale University from 1987 to 1991, and a postdoctoral researcher at the Robotics Research Group at University of Oxford, U.K., from 1991 to 1993. In 1993 she joined Bilkent University, Ankara, where she is currently an as- sociate professor in the Department of Electrical Engineering. Dr. Barshan is the founder of the Robotics and Sensing Laboratory in the same department. She is the recipient of the 1994 Nakamura Prize awarded to the most outstanding paper at the 1993 IEEE/RS Intelligent Robots and Systems International Conference; the 1998 TUBITAK Young Investigator Award; and the 1999 Mustafa N. Parlar Foundation Research Award. Dr. Barshan's current research interests include intelligent sensors, sonar and inertial navigation systems, sensor-based robotics, and multisensor data fusion. 